\title{
Panitumumab-Associated Drug- Induced Immune Thrombocytopenia in a Patient with Colorectal Cancer
}

\author{
Shigemasa Takamizawa ${ }^{a}$ Hirokazu Shojia Hidekazu Hirano ${ }^{a}$ Koji Izutsu ${ }^{\text {b }}$ \\ Shun Yamamoto ${ }^{a}$ Satoru Iwasa ${ }^{a} \quad$ Yoshitaka Honma $^{a}$ Natsuko Okita ${ }^{a}$ \\ Atsuo Takashima ${ }^{a}$ Ken Kato ${ }^{a}$ Narikazu Boku ${ }^{a}$ \\ aDepartment of Gastrointestinal Medical Oncology, National Cancer Center Hospital, Tokyo, \\ Japan; bepartment of Hematology, National Cancer Center Hospital, Tokyo, Japan
}

\section{Keywords}

Panitumumab · Drug-induced immune thrombocytopenia $\cdot$ Thrombocytopenia $\cdot$ Adverse event

\begin{abstract}
Severe thrombocytopenia is a rare adverse event of panitumumab. Here, we report the first patient with metastatic colorectal cancer who developed severe thrombocytopenia, diagnosed as panitumumab-associated drug-induced immune thrombocytopenia (DITP). A clinical diagnosis of DITP can be obtained by excluding other causes of thrombocytopenia and is confirmed by the recovery of thrombocytopenia after the discontinuation of the suspected drug. Treatment includes permanent discontinuation of the suspected drug. Re-exposure should be avoided. It should be kept in mind that panitumumab can induce DITP in the case of a new, sudden, unexpected, and isolated drop in platelet count after excluding other causes of thrombocytopenia.

\section{Introduction}

Panitumumab is an anti-epidermal growth factor receptor monoclonal antibody, approved for patients with wild-type KRAS metastatic colorectal cancer [1-3]. While erythema, pruritus, rash acneiform, and dry skin are major adverse events of panitumumab [2], severe thrombocytopenia is rare [4]. Here, we report a patient with metastatic colorectal cancer who developed severe thrombocytopenia, diagnosed as panitumumab-associated drug-induced immune thrombocytopenia (DITP). 


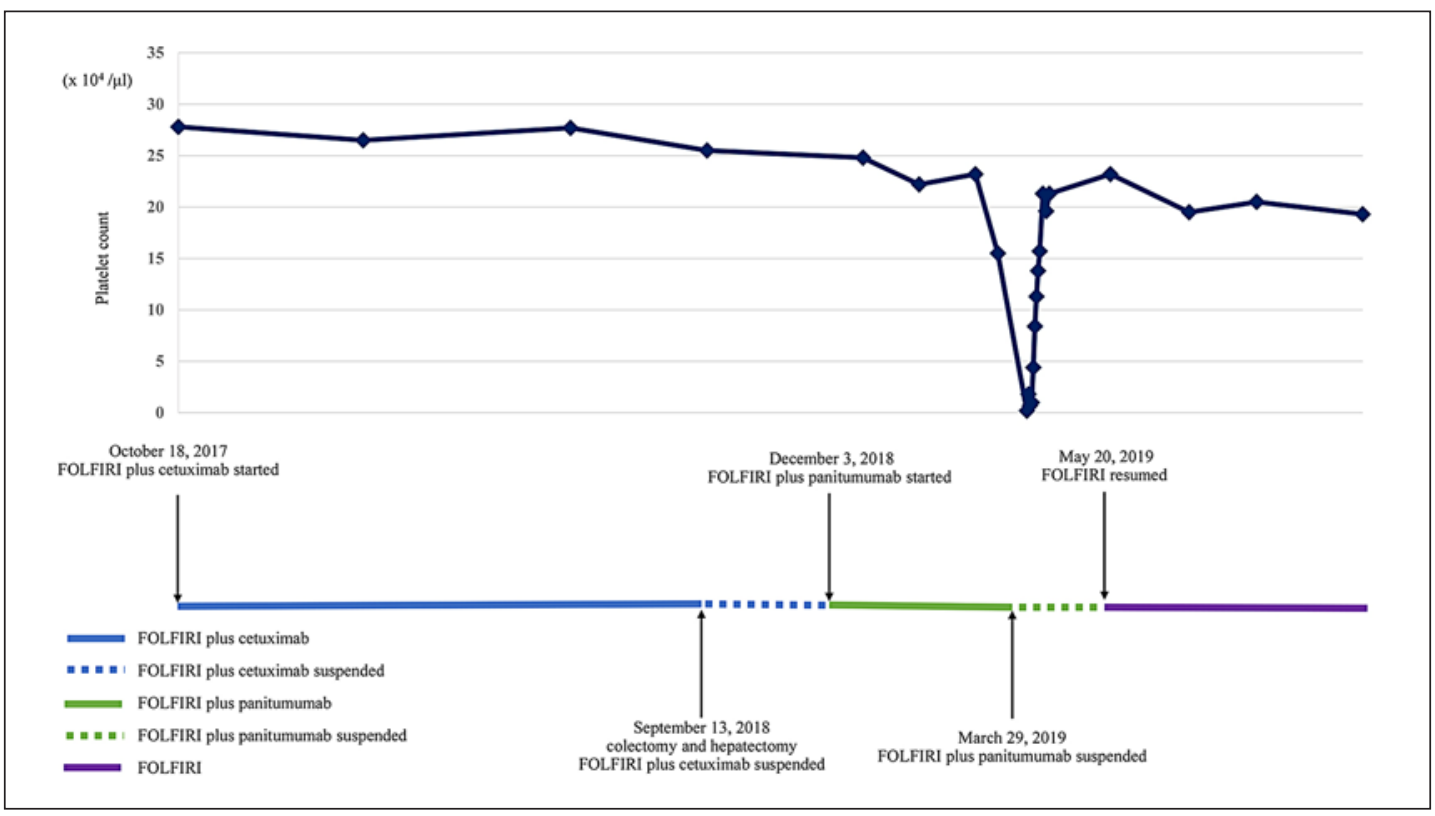

Fig. 1. Timeline of events and platelet count trend, from the initiation of irinotecan, L-leucovorin, and 5-fluorouracil (FOLFIRI) plus cetuximab. Arrows represent specific events as described.

\section{Case Presentation}

A 77-year-old female had transverse colon cancer with metastasis to the liver. Her medical history included hypertension, osteoporosis, and Helicobacter pylori infection with eradication therapy. The patient received FOLFIRI (irinotecan $150 \mathrm{mg} / \mathrm{m}^{2}$, L-leucovorin 200 $\mathrm{mg} / \mathrm{m}^{2}, 5$-fluorouracil $400 \mathrm{mg} / \mathrm{m}^{2}$ bolus infusion, and then $2,400 \mathrm{mg} / \mathrm{m}^{2}$ continuous infusion) every 2 weeks plus cetuximab (loading dose of $400 \mathrm{mg} / \mathrm{m}^{2}$, followed by $250 \mathrm{mg} / \mathrm{m}^{2}$ ) every week for 14 cycles from October 2017. Thrombocytopenia was not apparent during treatment with FOLFIRI plus cetuximab. Although the patient underwent a colectomy and hepatectomy after tumor shrinkage in September 2018, she experienced recurrence in the liver and bone metastasis 2 months later. Panitumumab was selected to reduce hospital visits, and FOLFIRI plus panitumumab (6 mg/kg) every 2 weeks was resumed from December 2018. The patient achieved an objective response without severe adverse events. After seven cycles, without an episode of recent infection, and the use of heparin, she experienced hematemesis. Upper gastrointestinal endoscopy revealed only atrophic gastritis with no findings of active bleeding.

Laboratory tests showed the following: hemoglobin, $7.5 \mathrm{~g} / \mathrm{dL}$; white blood cells, 4,300/ $\mu \mathrm{L}$; platelets, $0.2 \times 10^{4} / \mu \mathrm{L}$ (immature platelet fraction $20.8 \%$, reticulated platelets $67.7 \%$ ); prothrombin time, $13.5 \mathrm{~s}$; activated partial thromboplastin time, $26.4 \mathrm{~s}$, and D-dimer $0.7 \mu \mathrm{g} /$ mL. Fragmented red blood cells and hemolysis were not found. Bone marrow examination showed an increase in megakaryocytes with no findings of invasion by cancer cells. These results suggested increased consumption of platelets and excluded myelosuppression, hemophagocytic syndrome, disseminated intravascular coagulation, or thrombotic thrombocytopenic purpura. Contrast-enhanced computed tomography of the abdomen showed no splenomegaly.

The patient received platelet transfusions from days 1 to 4 in addition to the discontinuation of FOLFIRI plus panitumumab; however, her platelet count did not normalize. Hence, 
Takamizawa et al.: Panitumumab-Associated Drug-Induced Immune

Thrombocytopenia

since panitumumab-associated severe DITP was suspected, prednisolone $1.0 \mathrm{mg} / \mathrm{kg}$, from days 4 to 9 , and immunoglobulin $25 \mathrm{~g} /$ day, from days 4 to 6 , were administered. The platelet count normalized on day 9 , and the patient was discharged on day 16. Prednisolone was continued for 4 months and then tapered off. Only FOLFIRI was resumed, with 21 cycles administered until progression of liver metastases, followed by four cycles of FOLFOX. Thrombocytopenia had not recurred at the time of publication (shown in Fig. 1).

\section{Discussion}

DITP is characterized by an acute onset of thrombocytopenia, caused by immune mechanisms involving specific antibodies that recognize platelet membrane glycoproteins [5]. This pathogenesis is complex and several major mechanisms have been suggested [6]. In general, DITP is uncommon, with an incidence of 10 cases per 1,000,000 inhabitants per year [7]. More than 100 drugs have been reported as the cause of DITP in a database [8,9]. The drug classes that are most often associated with DITP are cinchona alkaloid derivatives (quinine, quinidine), sulfonamides, NSAIDs, anticonvulsants, disease-modifying antirheumatic drugs, and diuretics [7]. However, panitumumab has never been associated with DITP.

A diagnosis of DITP is determined by the detection of antibodies that bind to platelets only in the presence of drugs $[9,10]$, which is the gold-standard method [11]. In this regard, a limitation of this case is that we could not identify drug-dependent antibodies. However, the measurement of such antibodies is not widely available [12]. Alternatively, a clinical diagnosis of DITP can be obtained by excluding other causes of thrombocytopenia and is confirmed by the recovery of thrombocytopenia after the discontinuation of the suspected drug. Clinical criteria have been established, whereby a drug's culpability could effectively be identified as unlikely, possibly, probably, or definitely related to thrombocytopenia [13]. In this case, other causes for thrombocytopenia, such as myelosuppression, hemophagocytic syndrome, disseminated intravascular coagulation, or thrombotic thrombocytopenic purpura, might be excluded. The use of new drugs was not initiated for more than a year, except for panitumumab. Therefore, the sudden onset of thrombocytopenia was highly suspected due to panitumumab-associated DITP. The levels of evidence for a causal relation between the drug and thrombocytopenia was probable.

With regard to treatment of DITP, no standard therapeutic approach outside of permanent discontinuation of the suspected drug exists [14]. The platelet count has been reported to recover in about a week, only after drug discontinuation, in many cases [12]. However, in this case, thrombocytopenia did not recover after the discontinuation of FOLFIRI and panitumumab. The condition was so severe and associated with life-threatening bleeding that additional treatment was required. Even though the administration of steroids or immunoglobulins is controversial [5], these were administered, with the platelet count subsequently becoming normalized by day 9 . Because of the long half-life of panitumumab (about 5-9 days) [15], the platelet count did not recover soon after the discontinuation of panitumumab. Drugdependent antibodies can persist for many years, and re-exposure to the drug might lead to the recurrence of thrombocytopenia, hence, chemotherapy without panitumumab has been resumed without the recurrence of thrombocytopenia to date. The sudden onset of thrombocytopenia, no other causes for thrombocytopenia, a response to steroids and immunoglobulins, no newly added drugs except panitumumab, and no recurrence of thrombocytopenia after resuming all drugs except panitumumab strongly support a diagnosis of panitumumabassociated DITP.

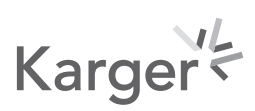




\section{Case Reports in Oncology}

\begin{tabular}{|c|c|}
\hline \multicolumn{2}{|c|}{ Case Rep Oncol 2021;14:85-89 } \\
\hline DOI: 10.1159/000512821 & $\begin{array}{l}\text { (c) } 2021 \text { The Author(s). Published by S. Karger AG, Basel } \\
\text { www.karger.com/cro }\end{array}$ \\
\hline
\end{tabular}

Takamizawa et al.: Panitumumab-Associated Drug-Induced Immune Thrombocytopenia

\section{Conclusion}

This rare case is the first report of panitumumab-associated DITP. Although DITP is a rare adverse event, its consequences may be severe and life-threatening. It should be kept in mind that panitumumab can induce DITP in the case of a new, sudden, unexpected, and isolated drop in platelet count after excluding other causes of thrombocytopenia.

\section{Statement of Ethics}

The Institutional Review Board approved the case report and submission of medical literature. We obtained written informed consent from the patient to publish her case.

\section{Conflict of Interest Statement}

Dr. Shoji reports other from ONO Pharmaceuticals, MSD, Astellas, and Amgen outside the submitted work. Dr. Izutsu reports grants and personal fees from Takeda outside the submitted work. Dr. Kato reports other from ONO, MSD, Shionogi, AstraZeneca, Merck Biopharma, and Beigene outside the submitted work. Dr. Boku reports grants and personal fees from Ono, grants from Takeda, and personal fees from Taiho and Bristol-Myers Squibb outside the submitted work. All other authors have nothing to disclose.

\section{Funding Sources}

There were no sources of funding related to this work.

\section{Author Contributions}

S.T. and H.S. wrote the manuscript; H.H. and K.I. suggested important intellectual content and took part in the critical discussion; S.Y., S.I., Y.H., N.O., A.T., K.K., and N.B. reviewed the manuscript; all authors read and approved the final version of the manuscript.

\section{References}

1 Douillard JY, Siena S, Cassidy J, Tabernero J, Burkes R, Barugel M, et al. Randomized, phase III trial of panitumumab with infusional fluorouracil, leucovorin, and oxaliplatin (FOLFOX4) versus FOLFOX4 alone as first-line treatment in patients with previously untreated metastatic colorectal cancer: the PRIME study. J Clin Oncol. 2010;28(31):4697-705.

2 Van Cutsem E, Peeters M, Siena S, Humblet Y, Hendlisz A, Neyns B, et al. Open-label phase III trial of panitumumab plus best supportive care compared with best supportive care alone in patients with chemotherapyrefractory metastatic colorectal cancer. J Clin Oncol. 2007;25(13):1658-64.

3 Köhne CH, Hofheinz R, Mineur L, Letocha H, Greil R, Thaler J, et al. First-line panitumumab plus irinotecan/5fluorouracil/leucovorin treatment in patients with metastatic colorectal cancer. J Cancer Res Clin Oncol. 2012; 138(1):65-72.

4 Petrelli F, Ardito R, Ghidini A, Zaniboni A, Ghidini M, Barni S, et al. Different toxicity of cetuximab and panitumumab in metastatic colorectal cancer treatment: a systematic review and meta-analysis. Oncology. 2018; 94(4):191-9.

5 Jardim DL, Rodrigues CA, Novis YA, Rocha VG, Hoff PM. Oxaliplatin-related thrombocytopenia. Ann Oncol. 2012;23(8):1937-42. 
6 Aster RH, Curtis BR, McFarland JG, Bougie DW. Drug-induced immune thrombocytopenia: pathogenesis, diagnosis, and management. J Thromb Haemost. 2009;7(6):911-8.

7 van den Bemt PM, Meyboom RH, Egberts AC. Drug-induced immune thrombocytopenia. Drug Saf. 2004; 27(15):1243-52.

8 George JN [Internet]. Single report database for drug-induced thrombocytopenia: an update 2018 [cited 2020 Oct 17]. University of Oklahoma Health Sciences Center. Available from: https://ouhsc.edu/platelets/singleditp/WEB\%20Table\%20Individual\%202018.pdf.

9 Arnold DM, Kukaswadia S, Nazi I, Esmail A, Dewar L, Smith JW, et al. A systematic evaluation of laboratory testing for drug-induced immune thrombocytopenia. J Thromb Haemost. 2013;11(1):169-76.

10 Bougie D, McFarland J, Curtis B, Aster R [Internet]. Drug-induced immune thrombocytopenia results of the testing for drug-dependent platelet-reactive antibodies by the Blood Center of Wisconsin [cited 2020 0ct 17]. University of Oklahoma Health Sciences Center. Available from: https://www.ouhsc.edu/platelets/WEB\%20 Table\%20Lab\%202018.pdf.

11 Laurichesse M, Pedrono M, Guilleray G, Gallienne L, Cherel M, Schmitt F, et al. Oxaliplatin and methylprednisolone-induced thrombocytopenia and monocytopenia, owing to anti-GPIIbIIIa and -CD36 antibodies in a patient with colorectal cancer. Clin Colorectal Cancer. 2020;19(4):e277-80.

12 George JN, Aster RH. Drug-induced thrombocytopenia: pathogenesis, evaluation, and management. Hematology Am Soc Hematol Educ Program. 2009;2009(1):153-8.

13 George JN, Raskob GE, Shah SR, Rizvi MA, Hamilton SA, Osborne S, et al. Drug-induced thrombocytopenia: a systematic review of published case reports. Ann Intern Med. 1998;129(11):886-90.

14 Stack A, Khanal R, Denlinger CS. Oxaliplatin-induced immune thrombocytopenia: a case report and literature review. Clin Colorectal Cancer. 2020.

15 Krens LL, Baas JM, Guchelaar HJ, Gelderblom H. Pharmacokinetics and safety of panitumumab in a patient with chronic kidney disease. Cancer Chemother Pharmacol. 2018;81(1):179-82. 